\title{
Performance Investigation and Enhancement of Fiber Bragg Gratingfor Efficient Sensing Measurement
}

\author{
Chaoui Fahd ${ }^{1}$, Aghzout Otman $^{2}$, EL Yakhloufi Mounir ${ }^{3}$ \\ ${ }^{I}$ Dept of Physics, Faculty of Sciences, UAE, Tetouan, Morocco \\ ${ }^{2}$ Electronics \& Microwaves Group, Telecommunication Dept, ENSA, UAE, Tetouan, Morocco \\ ${ }^{3}$ Condensed Matter Physics Group, Faculty of Sciences, UAE,Tetouan, Morocco
}

\begin{abstract}
In this paper, the performance of various windowfunctions for Fiber Bragg Grating Sensor (FBGS)is investigated and evaluated in order to get optimized reflection spectrum with high reflectivity and an efficient side lobe suppression for efficient sensing measurement applications.For this purpose, a wide range of design parameters which include grating length and refractive index modulation amplitudehas been chosen to evaluate the sensor design. The performances of the different windowfunctions have been then compared in terms of reflectivity, full width half maximum bandwidth (FWHM), and sidelobe level(SLL) so as to get the most suitable design parametersto be used for sensing measurement.The simulation results presented in this paper show the effectiveness of the optimizedFBG sensor, which can be further implemented for high performance sensing applications.
\end{abstract}

Keywords: Fiber Bragg Grating Sensor (FBGS), Full Width at Half Maximum (FWHM), Side Lobe Level (SLL)

\section{Introduction}

Over the last few decades, Fiber Bragg Grating Sensor (FBGS) has proven very successful in various sensing and monitoring applications in composites, civil engineering, aerospace, marine, oil \& gas, and smart structures [1]. In opposition to the conventional electrical sensors, FBG sensors assure immunity to electromagnetic fields, and can be easily installed at low cost to measure important parameters such as strain, temperature, pressure, vibration, acceleration, bending, torsion, [2][3]. The important feature of FBGS is that the sensed information is translated into a wavelength shift, giving reproducible measurements in spite of optical losses and intensity fluctuations. However, uniform apodization of FBGS leads to a reflection spectrum with large side lobes around the main reflected wavelength [4], which makes them unsuitable for distributed sensing systems. For this reason, apodized FBGS have been widely developed and applied in optical fiber technology as they have good filtering performance and high side lobe suppression. Concordantly, appropriate apodization results in high side lobe suppression and superior grating performance [5]. In this work, we investigate comprehensively the FBGS apodization performance based on Reflectivity level, Full-Width at Half Maximum (FWHM) and Side Lobe Level (SLL) across different values of grating length, refractive index modulation depth and different Apodization profiles in order to get optimized reflection spectrum with high reflectivity and efficient side lobe suppression. The rest of the paper is organized as follows: In Section II, we briefly describe the general theoretical model used for the calculation of the fiber Bragg Grating Reflection spectrum. The main apodization profiles considered in this paper are also presented in this section. In Section III, we compare the effect of the apodization profile on reflectivity, FWHM, and side lobe level of the sensor grating. The comparison is further quantified to find out the most suitable setting of the FBG sensor to be used in sensing measurement. The conclusions are given in Section IV

\subsection{Apodization and Reflectivity:}

\section{Theory And Modeling}

FBG, first demonstrated by Hill et al. [6], is developed by inscribing periodic refractive index modulation into the core of optical fiber using intense ultraviolet (UV) source through interferometric, point-bypoint or phase mask technique. The alteration of the core refractive index is possible because of the photosensitivity[7], a permanent change in the index refraction of the core when exposed to light, of the fiber optic. The magnitude of the refractive index change obtained from the UV exposure depends on several factors such as irradiation condition (wavelength, intensity), the composition of the core material, and pre-irradiation processing technique[8]. The variation of the refractive index change along the fiber axis determines the optical properties of FBGs. At the Bragg condition, part of the broadband light source launched into FBGs will be reflected as shown in Fig. 1. Resulted gratings reflect the propagated light in fiber according to Bragg wavelength which is given as follow: 


$$
\lambda_{B}=2 n_{e f f} \Lambda
$$

where $n_{\text {eff }}$ is the effective refractive index of the core of the fiber and $\Lambda$ is the grating period.The FBG is completely characterized by its refractive index distribution $n_{e f f}(z)$ along the fiber

$$
n_{e f f}(z)=n_{0}+f(z) \cdot \Delta n_{a c} \cdot v \cdot \cos \left(\left(\frac{2 \pi}{\Lambda}\right) z+\theta(z)\right)+\Delta n_{d c}
$$

where $z$ is the position, $n_{0}$ is the refractive index prior to grating inscription, $\Lambda$ is the grating period, $\Delta n_{a c}$ is the refractive index modulation amplitude, $\Delta n_{d c}$ is the average change in refractive index, $f(z)$ is the window function, $\theta(z)=\frac{2 \pi}{\Lambda} C z^{2}$ is the chirp function where $C$ is the chirp parameter, and $v$ is the fringe visibility [2][9].

\subsection{Coupled Mode Theory:}

Coupled Mode Theory (CMT) describes the behavior of Bragg grating, where the counter-propagating fields inside the grating structure are related by coupled differential equations. Transfer Matrix Method (TMM) will be used to solve coupled mode Equations for Bragg grating structure to obtain its spectral response.The electric field distribution along the grating of the backward and forward propagating waves is shown in Fig. 1 and can be expressed as

$$
\begin{aligned}
& E_{a}(z, t)=A(z) e^{i[\omega t-\beta z]} \\
& E_{b}(z, t)=B(z) e^{i[\omega t+\beta z]}
\end{aligned}
$$

where $A(z)$ and $B(z)$ are the slowly varying amplitudes of the backward and the forward traveling waves respectively and $\beta$ is the wave propagation constant [9].

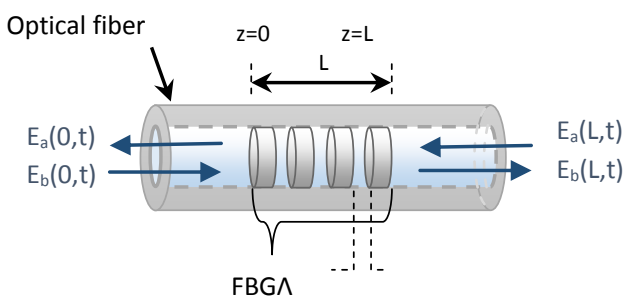

Fig. 1: The forward and backward propagating waves within the FBG

The coupled mode equations that describe the complex amplitudes $A(z)$ and $B(z)$ are given as follow:

$$
\begin{aligned}
& \frac{d A(z)}{d z}=i k B(z) e^{-i(2 \Delta \beta) z} \\
& \frac{d B(z)}{d z}=-i k^{*} A(z) e^{i(2 \Delta \beta) z}
\end{aligned}
$$

Where $\beta$ is the wave propagation constant, $\Delta \beta=\beta-\beta_{0}$ is the differential propagating constant $(\beta=$ $2 \pi n 0 \lambda ; \beta 0=\pi \Lambda$, andk is the coupling coefficient. For uniform gratings, $\mathrm{k}$ is a constant and it is related to the index modulation depth.For sinusoidal variations of the index perturbation the coupling coefficient $\mathrm{k}$ is given by:

$$
k(z)=\frac{\pi}{\lambda} \Delta n_{a c} \cdot f(z) \cdot v
$$

In this work, the well-known transfer matrix method is applied to solve the couple mode equations and to obtain the spectral response of the fiber grating.

\subsection{Transfer Matrix Method:}

In this approach, the grating is divided into $N$ uniform sections; each section is represented by a $2 \times 2$ matrix. By multiplying these matrices, a global matrix that describes the whole grating is obtained. Let the section length be $\Delta=L / N$. By applying the appropriate boundary conditions and by solving the coupled-mode equations, we find the following transfer matrix relation between the fields at $z$ and at $z+\Delta$. 


$$
\left[\begin{array}{l}
A(z) \\
B(z)
\end{array}\right]=\left[\begin{array}{cc}
\cosh (\gamma \Delta)-i \frac{\Delta \beta}{\gamma} \sinh (\gamma \Delta) & -i \frac{\mathrm{k}}{\gamma} \sinh (\gamma \Delta) \\
i \frac{\mathrm{k}}{\gamma} \sinh (\gamma \Delta) & \cosh (\gamma \Delta)+i \frac{\Delta \beta}{\gamma} \sinh (\gamma \Delta)
\end{array}\right]\left[\begin{array}{l}
A(z+\Delta) \\
B(z+\Delta)
\end{array}\right]
$$

Where $\gamma=\sqrt{\mathrm{k}^{2}-\Delta \beta^{2}}$ is phase shift to Bragg wavelength $\lambda_{B}$. We can connect the fields at the two ends of the grating through:

$$
\left[\begin{array}{l}
A(0) \\
B(0)
\end{array}\right]=T\left[\begin{array}{l}
A(L) \\
B(L)
\end{array}\right]
$$

Where

$$
T=T_{1} * T_{2} * \ldots T_{j} \ldots T_{N-1} * T_{N}
$$

As a result, $\mathrm{T}$ is a $2 \times 2$ matrix elements

$$
T=\left[\begin{array}{ll}
T_{11} & T_{12} \\
T_{21} & T_{22}
\end{array}\right]
$$

The reflectivity of the grating with constant modulation amplitude and period is defined by:

$$
R=\frac{T_{21}}{T_{11}}
$$

\subsection{Window functions:}

The mainwindow (apodization) functionsdiscussed in literature are [4][5][8]:

1- Uniform :

$$
f(z)=1
$$

2- Gaussian function :

$$
f(z)=\exp \left(-4 \cdot \ln (2) \cdot\left(\left(z-\frac{L}{2}\right) / s \cdot L\right)^{2}\right)
$$

3- Hamming function :

$f(z)=0.54-0.46 \cos (2 \pi z / L)$

4- Nuttal function: $\quad f(z)=a_{0}-a_{1} \cos (2 \pi(z / L))+a_{2} \cos (4 \pi(z / L))-a_{3} \cos (6 \pi(z / L))$

Where $a_{0}=0.3635819, a_{1}=0.4891775, a_{2}=0.1365995, a_{3}=0.0106411$ are the window coefficients, $L$ is the grating length, $Z$ is the coordinate of light propagation along the length of FBG and $s=0.5$ is taper parameter used for fine tuning of reflection spectrum[4].

\section{Results And Discussion}

The simulations are performed by assuming that the FBG is inscribed into standard telecommunication single mode optical fiber with $2 \mu \mathrm{m}$ core radius. Bragg wavelength is set to1550nm. $\Delta n_{a c}$ varies from $10^{4}$ to $5 \times 10^{4}$ while $L$ is maintained at $10 \mathrm{~mm}$. Then, the simulations are repeated with varying $L$ from $4 \mathrm{~mm}$ to $20 \mathrm{~mm}$ while $\Delta n_{a c}$ is maintained at $2 \times 10^{4}$. These values are chosen based on previous research papers[11, 12]. Optigrating (version 4.2) from Optiwave is used for simulation purpose. The windowfunction with high reflectivity and high side lobe suppression is then analyzed under strain sensor in order to get the better wavelength shift.

\subsection{Performance evaluation based on window function :}

Fig.2 shows the effect of apodization on the reflection spectrum for the different apodization profile discussed on this paper Uniform, Gaussian, Hamming and Nuttal respectively. It can be clearly seen that apodized gratings have superior filtering performance and high side lobe suppression compared to Uniform grating. However, it can also be observed that the apodization functions have less reflectivity than uniform grating due to apodization process. 

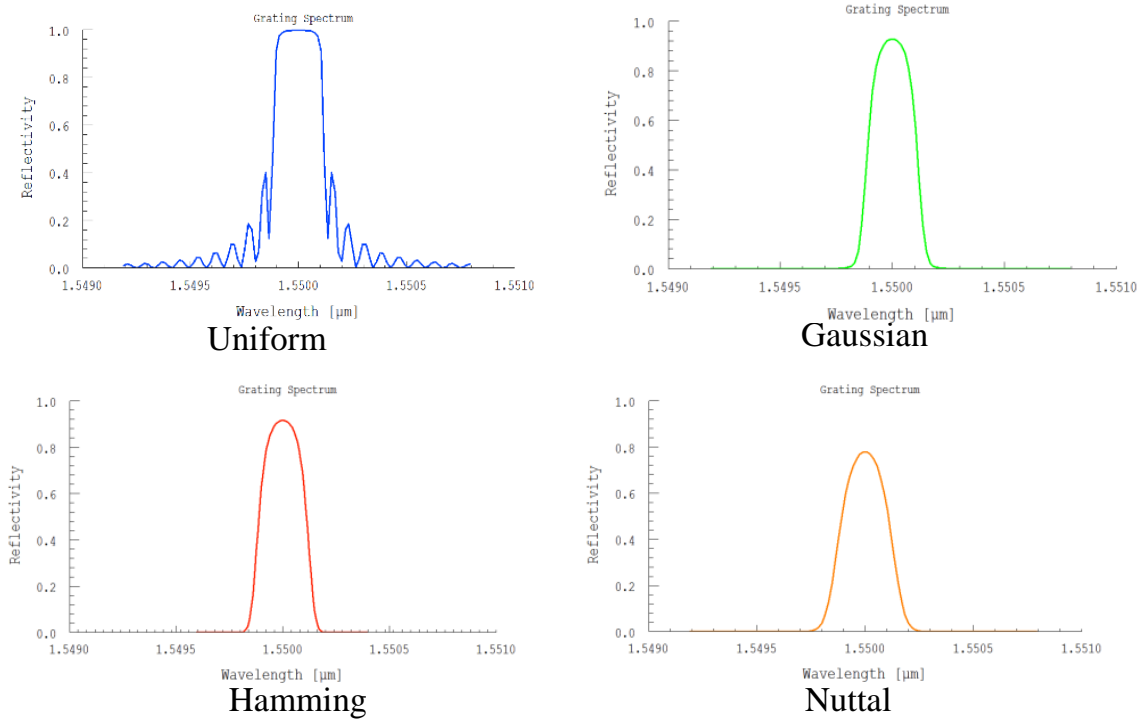

Fig. 2: Grating spectrum of the window functions

\subsection{Performance evaluation based on Refractive index modulation amplitude :}

The reflectivity as function of $\Delta n_{a c}$ while maintaining $\mathrm{L}$ at $10 \mathrm{~mm}$ for the different apodization profiles is depicted in Fig. 3. It can be observed that reflectivity increases as $\Delta n_{a c}$ increases for all the apodization profiles.It can also be noticed that theHamming profile provides the highest value, (Hamming and Gaussian curves are almost the same), whereas the Nuttal function provides the lowest reflectivity value among the simulated apodized FBGs.



Fig. 3:Reflectivity curves of the apodization profiles at $\mathrm{L}=10 \mathrm{~mm}$

Fig. 4 shows the FWHM and the sidelobe level versus $\Delta n_{a c}$ for the different apodization functions for a fixed value of grating length $(L=10 \mathrm{~mm})$. It can be seen that the higher the $\Delta n_{a c}$ the wider the FWHM, however the side lobe level is almost the samewithin each apodization function after a certain threshold $\left(2 \times 10^{-4}\right.$ for Gaussian and Hamming, $3 \times 10^{-4}$ for Nuttal). Moreover, for apodized gratingsa lower sidelobe level is noticed, as expected, compared to the uniform one. 

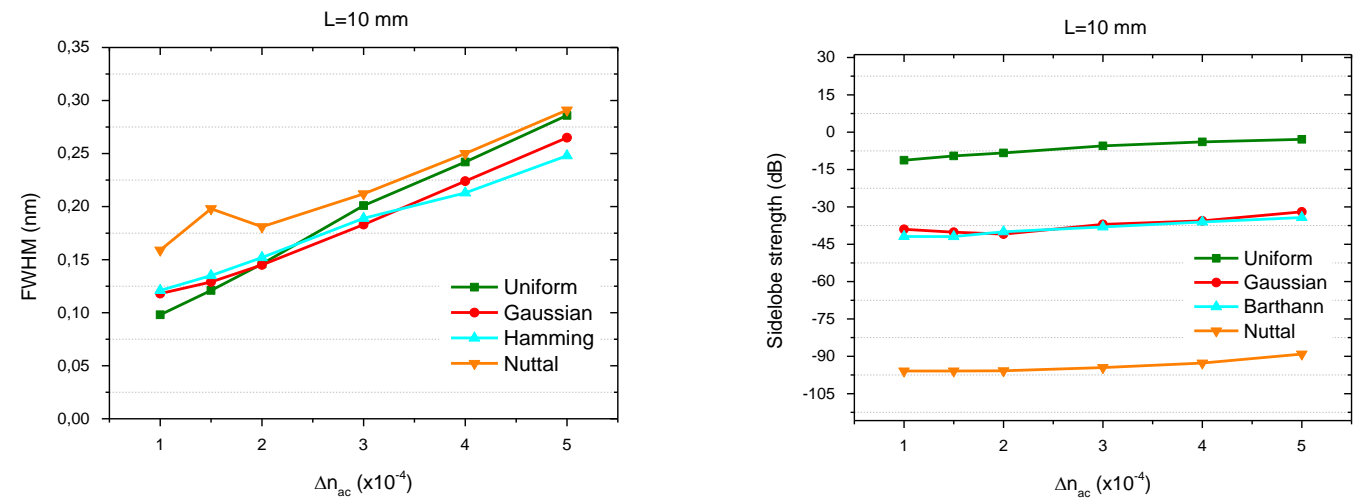

Fig. 4: FWHM and Sidelobe strength of the apodization profiles at $\mathrm{L}=10 \mathrm{~mm}$

\subsection{Performance evaluation based on Grating length :}

The reflectivity as function of $L$ while maintaining $\Delta n_{a c}$ at $2 \times 10^{-4}$ for the different apodization profiles is shown in Fig. 5. It is shown that reflectivity increases as Lincreases for all the apodization profiles. It can also be observed that Hamming profile again provides the highest value, whereas Nuttal provides the lowest reflectivity between all the apodized gratings.

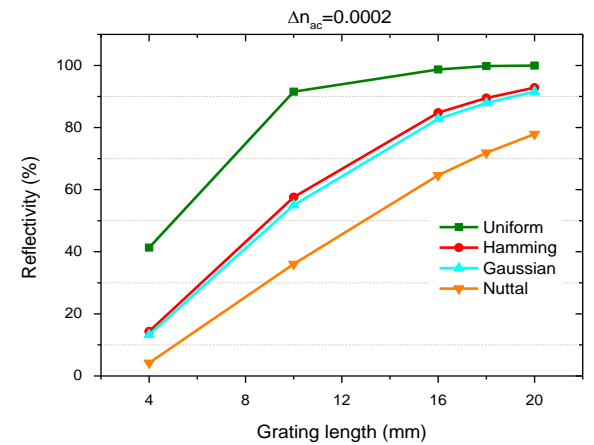

Fig. 5: Reflectivity curves of the apodization profiles at $\Delta n_{a c}=2 \times 10^{-4}$
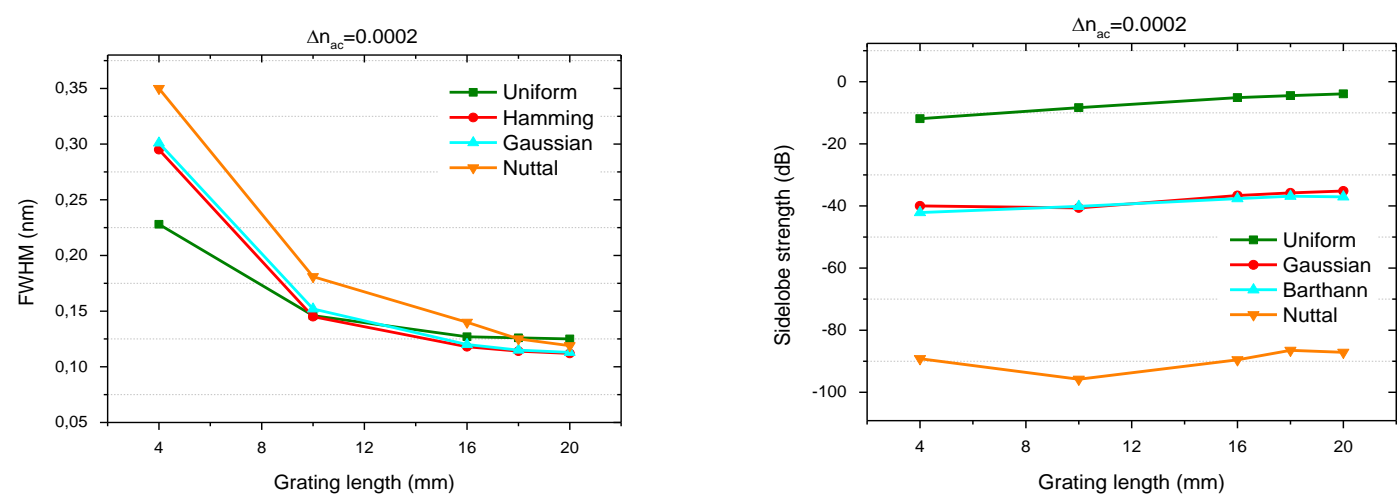

Fig. 6: FWHM and Sidelobe strength of the apodization profiles at $\Delta n_{a c}=2 \times 10^{-4}$

Fig. 6 shows the FWHM and the side lobe level versus grating length for a fixed value of refractive index modulation amplitude. It can be seen that the longer the grating the narrower the FWHM, while the side lobe level remains almost the same for Uniform, Gaussian, and Hamming after a certain threshold (10mm).

\subsection{Comparison summary:}

To choose the optimum reflection spectrum of the FBG sensor, performance comparison was carried out based on thresholds for Reflectivity, FWHM and Side lobe strength [4]:

- High reflectivity, with more than 50\%.

- Narrow FWHM, with less than $0.2 \mathrm{~nm}$. 
- $\quad$ Low Side lobe level, with less than $-25 \mathrm{~dB}$.

The previous thresholds are suggested with the aid of several sources, vendors and other literature reviews[4] [9].Table 1 shows the comparison summary of the different design parameters and the different apodization profiles. The comparison results show that Hamming apodization profile results in an overall superior performance as it provides high reflection with small FWHM and minimum side lob strength, compared with Uniform and Gaussian apodization. Nuttal profile offers an excellent side lobe suppression, but it wastes an important amount of the reflectivity power.

Table 1: Comparison summary of the different apodization functions

\begin{tabular}{|c|c|c|c|}
\hline Window function & Reflectivity & FWHM & Side Lobe \\
\hline Uniform & Excellent & Acceptable & Bad \\
\hline Gaussian & Very good & Good & Very good \\
\hline Hamming & Very good & Good & Very good \\
\hline Nuttal & Acceptable & Good & Excellent \\
\hline
\end{tabular}

One can conclude from the previous results that the use of uniform gratingin measurement sensing applications affects the reflectivity patterns of adjacent channels due to the high side lobe level. For this reason, it is quiet preferable to use only apodized FBG sensors in distributed sensing system in order to avoid the undesired influence of sidelobes on the adjacent channels. It can also be concluded that Hamming profile results in an overall superior performance as it provides high reflection with small FWHM and minimum side lobe strength, compared with Nuttal and Gaussian apodizations.

\section{Conclusion}

In this paper, the performances of Fiber Bragg Gratingfor differentwindow profiles have been compared in terms of reflectivity, FWHM bandwidth, and side lobe suppression to get the most suitable design parameters to be used for accurate sensing measurement. The simulation results shows that the use of uniform grating in measurement sensing applications affects the reflectivity patterns due to the high side lobe level. For this reason, it is quiet preferable to use only apodized FBG sensors in distributed sensing system in order to avoid the undesired influence of side lobes on the adjacent channels. Hamming apodization profile results in an overall superior performance as it provides high reflection with small FWHM and minimum side lobe strength, compared with Uniformand Gaussian apodizations.Nuttal profile offers an excellent side lobe suppression, but it wastes an important amount of reflectivity power.

\section{References}

[1]. Frantisek Urban, Jaroslav Kadlec and Radek Vlach, "Design of a Pressure Sensor Based on Optical Fiber Bragg Grating Lateral Deformation," sensors, pp. 11212-11225, 2010.

[2]. Karin Ennser, Mikhail N. Zervas "Optimization of Apodized Linearly Chirped Fiber Gratings for Optical Communications," IEEE JOURNAL OF QUANTUM ELECTRONICS, vol. 34, no. 5, MAY 1998.

[3]. B.A. Tahir, J. Ali, R.A. Rahman, "Strain measurements using fibre Bragg grating sensor," Am. J. Applied Sciences (Special Issue), no. 1546-9239., pp. 40-48, 2005.

[4]. Nazmi A. Mohammed, Taha A. Ali, Moustafa H. Aly, "Evaluation and performance enhancement for accurate FBG temperature sensor measurement with different apodization profiles in single and quasi-distributed DWDM systems," ELSEVIER- Optics and Lasers in Engineering 55, pp. 22-34, 2014.

[5]. M. J. Moghimi, H. Ghafoori-Fard, and A. Rostami, "Analysis and design of all-optical switching in apodized and chirped Bragg gratings," Progress In Electromagnetics Research B,, vol. 8, pp. 87-102, 2008.

[6]. K.O. Hill, B. Malo, F. Bilodeau, D.C. Johnson and J. Albert,, "Photosensitivity on optical fiber waveguides: application to reflection filter fabrication," Applied Physics Letter, vol. 32, no. 1978, pp. 747-749.

[7]. K. O. Hill and G. Meltz, "Fiber Bragg Grating Technology Fundamentals and overview," IEEE Journal of Lightwave Technology, vol. 15, p. 1263-1276, 1997.

[8]. Ian Yulianti, Abu Sahmah M. Supa'at, Sevia M. Idrus, Abdulaziz M. Al-hetar, "Simulation of Apodization Profiles Performances for unchirped Fiber Bragg Gratings," IEEE 978-1-4244-7187-4, 2010.

[9]. Herber Venghaus, "Venghaus H.Wavelength filters in fibre optics, wavelength filters in fibre optics," Springer (Chapter 5) 3-540$31769-4$.

[10]. G. Meltz, W.W. Morey, "Bragg grating formation and germanosilicate fiber photosensitivity," in Intern. Workshop on Photo induced Self-Organization Effects in Optical Fiber, Quebec, 1991.

[11]. Ugale S.P., Mishra V., "Optimization of fiber Bragg grating length for maximum reflectivity," Procedeeing of the International Conference on Communications and Signal Processing (ICCSP), IEEE, pp. 28-32, 2011. 